\title{
Temporal stability and absolute composition issues in molecular beam epitaxy of AlGaAs/GaAs THz QCL
}

\author{
I. S. Vasil'evskii ${ }^{1}$, A. N. Vinichenko ${ }^{1}$, M. M. Grekhov ${ }^{1}$, V. V. Saraykin ${ }^{2}$, A. N. Klochkov ${ }^{2}$, \\ N. I. Kargin ${ }^{1}$, R. A. Khabibullin' ${ }^{2}$, S. S. Pushkarev ${ }^{2}$ \\ ${ }^{1}$ National Research Nuclear University «MEPhI», Moscow, Russia, ivasilevskii@ mail.ru \\ ${ }^{2}$ V.G. Mokerov Institute of Ultra High Frequency Semiconductor Electronics of RAS, Moscow, Russia
}

The operation of the terahertz quantum cascade lasers (THz QCL) is strongly dependent on the repeatable fabrication of the well and barrier layers with the certain thicknesses throughout the whole active region stack epitaxial growth. This emphasizes the importance of the strict control of the growth rates and the stability of Si and group III effusion cell fluxes during growth [1]. It was reported [2] that two THz QCLs based on nominally identical multilayer heterostructures $\mathrm{Al} 0.15 \mathrm{Ga} 0.85 \mathrm{As} / \mathrm{GaAs}$ emit at the different frequencies of 2.59 and $2.75 \mathrm{THz}$ because of unintentional small deviations in the GaAs and AlAs growth rates (4 and $1.6 \%$ respectively). Authors [3] determined the thickness tolerance for working lasing heterostructures to be minimally above $2 \%$ while the structures with thickness deviation 4.3 and $6.5 \%$ are not lasing. In [4] the $\mathrm{Ga}$ cell temperature has to be increased to maintain a GaAs constant growth rate while the $\mathrm{Al}$ cell temperature remains nearly constant because variation in AlAs growth rate can be neglected. By using such growth rate compensation technique the two nominally identical structures approximately $10 \mathrm{mkm}$ overall thick were found to show thickness difference of $\sim 1 \%$. Thus calibration procedures and accurate analysis techniques become crucial to provide enhanced metrology possibilities.

In this work we discuss the following problems which have to be solved within the QCL growth metrology: precise determination of $\mathrm{Al}$ content in $\mathrm{Al}_{x} \mathrm{Ga}_{1-x} \mathrm{As}$ ternary, composition profiling, robust and rapid express-analysis for $\mathrm{AlGaAs} / \mathrm{GaAs}$ quantum well (QW) layer thickness and composition, cell regime corrections for proper III fluxes stabilization.

The origins of III group flow long-term drift are material rearrangement and depletion in the effusion cells and thermometry drift. The latter can arise if environmental temperature is changed and thermocouple loop hasn't reference junction.

For the absolute $\mathrm{Al}_{\mathrm{x}} \mathrm{Ga}_{1-\mathrm{x}} \mathrm{As}$ composition measurement the different techniques have been used: $\mathrm{X}$ ray diffractometry (XRD), photoluminescence spectroscopy (PL), secondary ion mass spectroscopy (SIMS), and Rutherford ion backscattering (RBS). SIMS measurements performed in cluster ion mode with $\mathrm{CsMe}^{+}$provide good accuracy for relative composition variation. Absolute content determination by different techniques, however, gives spreading within $\Delta x \sim \pm 0.015$. Discrepancy for XRD techniques $x$ determination arises from inaccuracy of $C_{11}$ and especially $C_{12}$ elastic coefficient for AlAs in the different databases. RBS are known as the most direct technique but it ensures absolute value within $\Delta x \sim \pm 0.01$ because Al haven't resolved separately but matches the area of GaAs substrate signal, so areal fit used rather than peak fit in this case. PL technique is quite sensitive $[5,6]$ but $E_{g}(x)$ dependencies have notable differences within $\Delta x \sim \pm 0.01$. PL is also can produce complicated spectrum due to doping and possible defects in $\mathrm{Al}_{x} \mathrm{Ga}_{1-x} \mathrm{As}$ (Fig. 1).

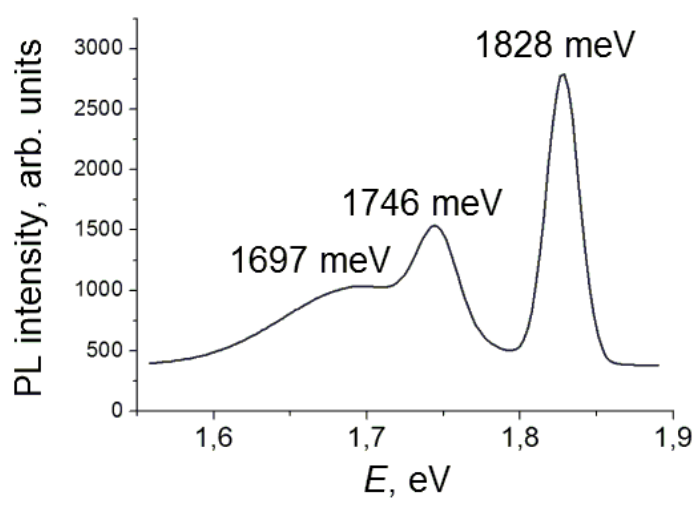

Fig. 1. PL spectra of $\mathrm{Al}_{x} \mathrm{Ga}_{1-x} \mathrm{As}$ at $T=77 \mathrm{~K}$

Series of experiments performed on Compact $21 \mathrm{~T}$ epitaxy on $2 \mu \mathrm{m}$ thick slightly $\mathrm{Si}$ doped $\mathrm{Al}_{x} \mathrm{Ga}_{1}$ ${ }_{x}$ As (structure \# 80) reveal a valuable drift of Al composition up to $1.2 \% \cdot \mu \mathrm{m}^{-1}$ for the range of $x \sim 0.15-$ 0.24 . After long-term cell regime stabilization and termal isolation of rear thermocouple terminals the variation has been decreased to $\sim 0.1 \% \cdot \mu \mathrm{m}^{-1}$ (Fig. 2), that could be sufficient for whole QCL structure growth.

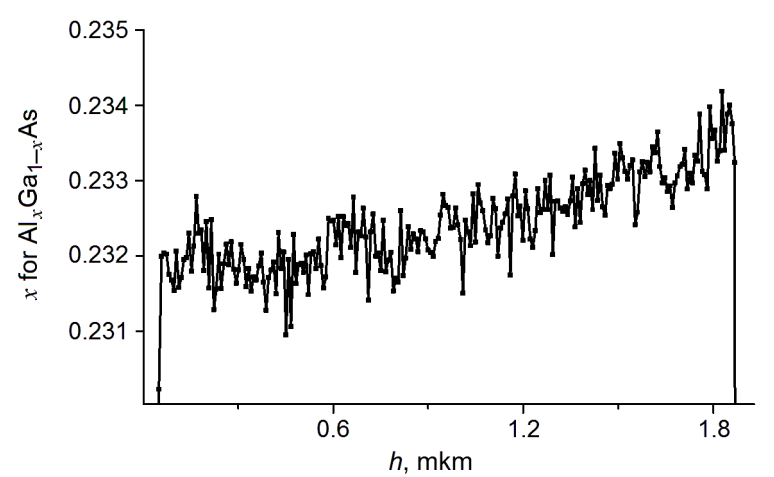

Fig. 2. $\mathrm{Al}$ content profile of the thick $\mathrm{Al}_{x} \mathrm{Ga}_{1-x} \mathrm{As}$ measured by SIMS

Another issue arises for superlattice structures $\mathrm{Al}_{x} \mathrm{Ga}_{1-x} \mathrm{As} / \mathrm{GaAs}$, where both thickness and composition precision are needed. We have confirmed high efficiency of the calibration structures containing both $\mathrm{Al}_{x} \mathrm{Ga}_{1-x}$ As layer and $\mathrm{Al}_{x} \mathrm{Ga}_{1-x} \mathrm{As}(96 \AA) / \mathrm{GaAs}(85 \AA)$ short-period superlattice (SPSL) for the combination of metrological techniques. After proper calibration of composition and layer thicknesses, easy and fast anal- 
ysis becomes possible by express technique as room temperature PL (Fig. 3). Sample \# 486 has additional bulk $\mathrm{Al}_{x} \mathrm{Ga}_{1-x}$ As layer that produces peak at $1.624 \mathrm{eV}$. Band structure calculation performed by Nextnano with conventional semiconductors parameters [7] shows good compliance with experiment. The technique sensitivity is about $0.6 \mathrm{meV}$ energy shift per $1 \AA$ GaAs thickness variation (Fig. 4). The absolute thickness determination accuracy for SPSL sample may be sufficiently higher by using XRD due to multiple satellite peaks and reaches $0.02 \AA$.

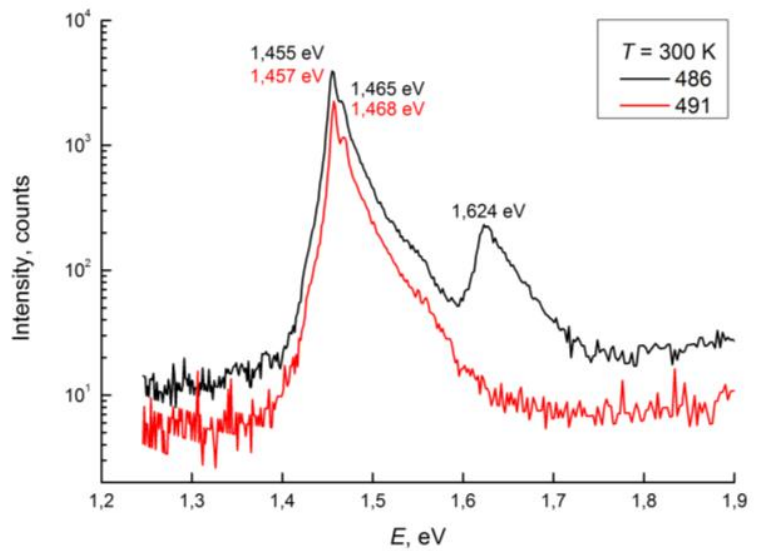

Fig. 3. Room temperature PL spectra of SPSL $\mathrm{Al}_{x} \mathrm{Ga}_{1-}$ ${ }_{x}$ As/GaAs samples

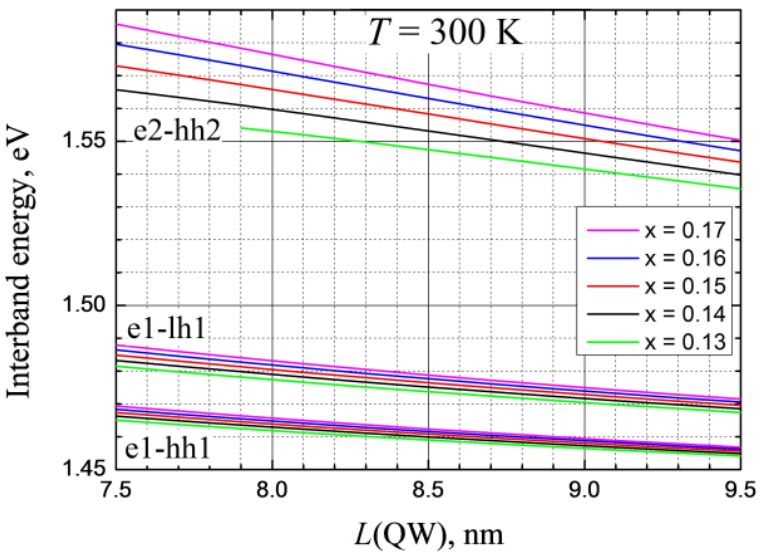

Fig. 4. Nomogramm of interband optical transitions in SPSL $\mathrm{Al}_{x} \mathrm{Ga}_{1-x}$ As $(96 \AA) / \operatorname{GaAs}\left(L_{Q W}\right)$

Low temperature photoluminescence spectra of the two similar SPSL structures (samples \# 486 and \# 491) shown on Fig. 5 reveal excellent matching of e1-lh1 and e1-hh1 transitions in the different structures at $1.54-1.55 \mathrm{eV}$. PL measurements demonstrate high intensity of transitions and narrow well-resolved exciton peaks. The peaks beyond the quantum well energy correspond to GaAs bulk exciton $(1.515 \mathrm{eV})$ and phonon replica of GaAs fundamental transition $(1.473 \mathrm{eV})$. The hidden peak at $1.63-1.64$ seeming as shoulder may be caused by e2-hh2 transition. Photoluminescence analysis based on e2-hh2 transition energy determination is more accurate in comparison with e1-transitions mainly in regard to $x$ estimation, as one may see from Fig. 4. The explanation is that e2 energy level position is more sensitive to the height of quantum well barrier which depends on $\mathrm{Al}_{x} \mathrm{Ga}_{1-x} \mathrm{As}$ content.

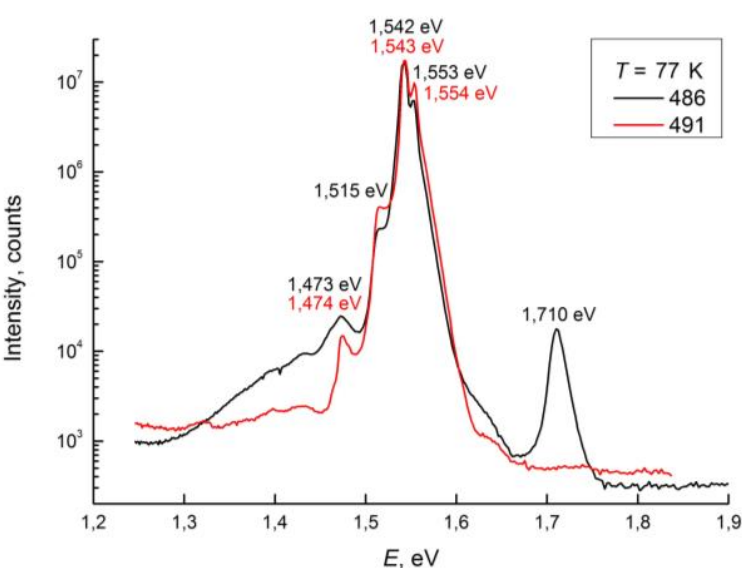

Fig. 5. Low temperature PL spectra of SPSL $\mathrm{Al}_{x} \mathrm{Ga}_{1}$ ${ }_{x} \mathrm{As} / \mathrm{GaAs}$ samples at $T=77 \mathrm{~K}$

As a result, different analysis techniques had been applied for $\mathrm{Al}_{x} \mathrm{Ga}_{1-x} \mathrm{As}$ composition analysis and spatial profile variation. XRD and PL techniques are collated and accuracy of the both are determined on short period $\mathrm{GaAs} / \mathrm{Al}_{x} \mathrm{Ga} \mathrm{1}_{-x} \mathrm{As}$ superlattice samples with layer arrangement, providing robust and rapid further control for QCL growth runs.

The study was partially supported by the Russian Federation President's Council on Grants (project MK-2342.2017.2) and by RFBR according to the research project № 17-02-00070 A.

\section{References}

1. Beere, H. E., Fowler, J.,C., Alton, J., Linfield, E.,H., Ritchie, D. A., Köhler, R., Tredicucci, A., Scalari, G., Ajili, L., Faist, J., Barbieri, S. MBE growth of terahertz quantum cascade lasers // Journal of Crystal Growth. 2005. V. 278. P. 756-764.

2. Khanna, S. P., Chakraborty, S., Lachab, M., Hinchcliffe, N. M., Linfield, E. H., Davies, A. G. The growth and measurement of terahertz quantum cascade lasers // Physica E. 2008. V. 40. P. 1859-1861.

3. Roch, T., Andrews, A. M., Fasching, G., Benz, A., Schrenk, W., Unterrainer, K., Strasser, G. High-quality MBE growth of $\mathrm{Al}_{x} \mathrm{Ga}_{1-x} \mathrm{As}$-based $\mathrm{THz}$ quantum cascade lasers // Central European Journal of Physics. 2007. V. 5, No. 2. P. 244-251.

4. Li, L. H., Zhu, J. X., Chen, L., Davies, A. G., Linfield, E. $H$. The MBE growth and optimization of high performance terahertz frequency quantum cascade lasers // Optics Express. 2015. V. 23, No. 3. P. 2720-2729.

5. Galiev, G.B., Klimov, E.A., Klochkov, A.N., Lavruhin, D.V., Pushkarev, S.S., Maltsev, P.P., Vasil'evskii, I.S. Specific features of the photoluminescence of HEMT nanoheterostructures containing a composite InAlAs/InGaAs/InAs/InGaAs/InAlAs quantum well // Semiconductors. 2015. V. 49, No. 2. P. 234-241.

6. Lavrukhin, D. V., Khabibullin, R. A., Ponomarev, D. S., Maltsev, P. P. Photoluminescence of heterostructures containing an $\operatorname{In}_{x} \mathrm{Ga}_{1-x}$ As quantum well with a high in content at different excitation powers // Semiconductors. 2015. V. 49, No. 9. P. 1218-1221.

7. Vurgaftman, I., Meyer, J. A., Ram-Mohan, L. A. Band parameters for III-V compound semiconductors and their alloys // Journal of Applied Physics. 2001. V. 89, No. 11. P. 5815-5875. 